\title{
Optimization of Multiple Receivers Solar Power Tower systems
}

\author{
E. Carrizosa ${ }^{\mathrm{a}, \mathrm{b}}$, C. Domínguez-Bravo ${ }^{\mathrm{b}, *}$, E. Fernández-Cara ${ }^{\mathrm{b}, \mathrm{c}}$, M. Quero $^{\mathrm{d}}$ \\ ${ }^{a}$ Department of Statistics and Operations Research, University of Seville, Spain \\ ${ }^{b}$ Mathematics Institute of the University of Seville, Spain \\ ${ }^{c}$ Department of Partial Equations and Numerical Analysis, University of Seville, Spain \\ ${ }^{d}$ Abengoa Solar New Technologies, Seville, Spain
}

\begin{abstract}
In this article a new procedure to optimize the design of a Multiple Receivers Solar Power Tower system is presented. The proposed procedure allows to optimize the different receivers (height, aperture tilt angle, azimuth angle and aperture size) as well as the heliostat field layout, seeking to minimize the levelized cost of thermal energy.

The optimization problem is high dimensional, with a black-box nonconvex objective function that is hard to compute.

Our method is based on an alternating greedy-based heuristic method, already used by the authors to design a system with a single receiver, which simultaneously optimizes the receivers and the heliostat field. The proposed procedure allows one to determine the overall number of heliostats, their locations and the aiming region of each field.
\end{abstract}

Keywords: solar thermal power, heliostat field layout, nonconvex optimization, greedy algorithm, multiple receivers

\section{Introduction}

Solar Power Tower (SPT) systems are known as one of the most promising technologies for producing solar electricity, as claimed in the literature $[2,21$, 25]. An SPT system is here considered to consist of three main components: tower, multiple receivers and a field of heliostats comprising rectangular mirrors. Direct solar radiation is reflected and concentrated by the heliostat field onto the receivers, placed at the top of the tower. The heliostats have two-axis movement

\footnotetext{
* Corresponding author.

Instituto de Matemáticas de la Universidad de Sevilla (IMUS)

Edificio Celestino Mutis- 1 Planta- D10.5

Avda. Reina Mercedes, s/n, 41012 Sevilla, Spain.

Tel: +34955420870 .

Email address: carmenanadb@us.es (C. Domínguez-Bravo)
} 
in order to reflect the direct light from the sun to fixed targets on the receivers. The thermal energy is transferred in the receivers to a heat transfer fluid and electricity is produced through a conventional thermodynamic cycle.

In recent years, higher power requirements are imposed on the SPT systems, calling for large-scale plants such as Gemasolar (19.9 MW and 2650 hel. see [33, 10]), Khi Solar One (50 MW and 4120 hel. see [35]) and Ivanpah (377 MW and 173500 hel. see $[34,6])$. Having only one receiver forces to locate heliostats far from the tower, increasing atmospheric and spillage losses, as pointed out in [24, 29, 32]. As said in [26], the experience shows that higher conversion efficiency of solar energy to electricity can be achieved only at the high temperatures reached using multiple receivers technology, as addressed in this paper.

There exist different proposals in the literature for spatial configurations of multiple receivers: vertical [28], circular [7, 14, 15], same focal spot [9] and horizontal, see [29, 31] and Figure 1. In our approach a horizontal distribution is considered where each receiver is characterized by its own height in the tower, aperture tilt angle, azimuth angle and aperture radius.

Different approaches of the heliostat field design have also been studied in the literature, see $[4,24,26,28,29,31]$. A common approach, called the field separation method, is to distinguish for each receiver a separate region, called aiming regions, where the heliostats will we placed, see [4]. Different shapes are usually imposed to the aiming regions, such as concentric circular trapezoids [28] and ellipses [29, 31]. However such aiming regions overlap, and it is not trivial how to decide the aiming strategy for heliostats at the intersection of the regions.

The use of the field separation method is mainly based on two facts: the different heliostats performance regarding their position in the field [32], and the computational time reduction by implementing simplified methods to calculate shading and blocking effects $[18,27]$.

If, for instance, we consider three aiming regions namely, North, West and East, the West region will be most efficient at the beginning of the day, and the East region in the afternoon. These different performances imply that the optimal number and density of heliostats will not necessarily be the same for each selected region.

The heliostat location problem is usually solved by applying a parameterized geometrical pattern. The pattern parameters are optimized and the final number of heliostats is obtained by oversizing the field, see [29]. This way, although the optimal parameters for the oversized field were obtained, there is a high risk that a strong distortion exists between the original and final configuration. The field separation strategy has already been used under radially-staggered layouts in [32].

In order to design the SPT system it is essential to understand the performance of the subsystems formed by the receivers and the heliostat field. The field and the receivers are interdependent, as pointed out in [28], where it is studied how increasing the receiver heights reduces some optical losses (shading, blocking and cosine effects) in large heliostat fields. It is thus important to design both components simultaneously.

In this article a new procedure to design a multiple receivers SPT system is 
presented, where the receivers and the heliostat field layout are simultaneously optimized. The receivers variables and the heliostats (number and positions) are optimized through an alternating process to obtain a multiple receivers system that minimizes the levelized cost of thermal energy (LCOE).

The methodology presented to solve the heliostat location problem is a greedy-based algorithm which follows a pattern free layout (used with single receiver systems in [12]). The final number of heliostats and the field shape are found during the optimization process. For simplicity, the heliostats are considered aiming the same receiver regardless the instant of time.

As far as the authors are aware of, the use of non imposed field geometries and the optimization of the field shape simultaneously with the receivers characteristics remains unexplored.

The rest of the paper is organized as follows. In Section 2, the SPT system is presented. Section 3 explains the optimization problem and our methodology to solve it. We apply the proposed algorithms to a given configuration, and discuss the main results in Section 4. The last section is devoted to summarize our results and to present some perspectives for further work.

\section{Decision Variables and Functions}

The optimal design of an SPT system consists of determining the apertures dimensions and receivers positions in the tower (Multiple Receivers Optimization) and the location of the heliostats (Field Optimization) so as to minimize the LCOE. In the following subsections the variables, feasible sets and functions involved in the optimization problem will be presented.

\subsection{Decision variables}

In the chosen system of coordinates, the positive $x$ axis is the North direction, the positive $y$ axis is the West direction and the $z$ axis is orthogonal to the ground. We will deal with cavity receivers with circular aperture, see $[2,13]$ and Figure 1(a). Although our approach is valid for any number of receivers, we consider for simplicity three receivers, called North, West and East, numbered as receiver 1, 2 and 3 respectively.

The four most relevant variables associated to each receiver design are considered, namely the height $h$ in the tower, the aperture tilt angle from the vertical $\xi$, the azimuth angle $\alpha$ which mesures the separation from the North axis and the aperture radius $r$, see Figures 1(a)-1(b). From now on we will denote by $\Theta_{i}$ the optimization variables related with receiver $i$ and by $\Theta$ the full list of decision variables concerning the receivers,

$$
\Theta=\left(\Theta_{1}, \Theta_{2}, \Theta_{3}\right) \in \mathcal{M}_{4 \times 3} \text { with } \Theta_{i}=\left(h_{i}, \xi_{i}, \alpha_{i}, r_{i}\right)^{t} \in \mathbb{R}^{4} \quad \forall i=1,2,3 .
$$

Some constraints, influenced by technical and legal regulations, determine the feasible region $\boldsymbol{\Theta}$. They can be written as follows:

$$
\boldsymbol{\Theta}=\left\{\begin{array}{ll}
\Theta \in \mathcal{M}_{4 \times 3}: \quad r_{\min } \leq r_{i} \leq \min \left(h_{i}, r_{\max }\right) \leq h_{\max } \quad \forall i=1,2,3 \\
& \xi_{i} \in[0, \pi / 2] \\
& \alpha_{i} \in\left[\underline{\alpha}_{i}, \bar{\alpha}_{i}\right]
\end{array}\right\},
$$


where $r_{\min }$ and $r_{\max }$ denote the minimum and a maximum receiver radius and $h_{\max }$ is the maximum value for the tower height. The ranges for the variables $\alpha_{i}$ are calculated as follows:

$$
\begin{array}{ll}
\underline{\alpha}_{1}=\max \left\{-\pi / 2, \alpha_{3}+\varsigma_{3}+\varsigma_{1}\right\}, & \bar{\alpha}_{1}=\min \left\{\pi / 2, \alpha_{2}-\varsigma_{2}-\varsigma_{1}\right\}, \\
\underline{\alpha}_{2}=\max \left\{0, \alpha_{1}+\varsigma_{1}+\varsigma_{2}\right\}, & \bar{\alpha}_{2}=\min \left\{\pi, \alpha_{3}-\varsigma_{3}-\varsigma_{2}\right\}, \\
\underline{\alpha}_{3}=\max \left\{-\pi, 2 \pi+\alpha_{2}+\varsigma_{2}+\varsigma_{3}\right\}, & \bar{\alpha}_{3}=\min \left\{0, \alpha_{1}-\varsigma_{1}-\varsigma_{3}\right\} .
\end{array}
$$

where the angles $\varsigma_{i}$ can be calculated through the following equations:

$$
\varsigma_{i}=\arcsin \left(\frac{r_{i}}{\sqrt{r_{i}^{2}+d_{\mathrm{ap}}^{2}}}\right) \quad \forall i=1,2,3 .
$$

The fixed parameter $d_{\mathrm{ap}}$ denotes the distance between the aperture and the center of coordinates, see Figures 1(a)-1(c).

In what concerns the field, the heliostats locations, given by the coordinates $(x, y)$ of their centers, are the variables to be used. All heliostats are assumed to be rectangular, to have the same dimensions and to be composed of rectangular facets. See $[11,20]$ for approaches where heliostats of different sizes are allowed. The finite collection of coordinates of the centers of the heliostats defines the heliostat field, $\mathcal{S}$. The heliostats must be located within a given region $\Omega \subset \mathbb{R}^{2}$ and they have to rotate freely avoiding collisions between them. The feasible region $\mathscr{S}$ can be written as follows:

$$
\mathscr{S}=\left\{\begin{array}{ll}
\mathcal{S} \subset \Omega: \quad|\mathcal{S}|<+\infty \\
& \left\|(x, y)-\left(x^{\prime}, y^{\prime}\right)\right\| \geq \delta \quad \forall(x, y),\left(x^{\prime}, y^{\prime}\right) \in \mathcal{S} \\
& (x, y) \neq\left(x^{\prime}, y^{\prime}\right)
\end{array}\right\},
$$

where $\delta>0$ is a given positive parameter, called security distance. Observe that the variable $\mathcal{S}$ is a set whose cardinality is not fixed in advance.

As already mentioned, we assume that each heliostat is always aiming the same receiver. The separate heliostat fields denoted by $\mathcal{S}_{i}$ for $i=1,2,3$, can be expressed as follows:

$$
\mathcal{S}_{i}=\{(x, y) \in \mathcal{S}: \text { heliostat at }(x, y) \text { aims at receiver } i\} \text { with } \mathcal{S}=\bigcup_{i=1}^{3} \mathcal{S}_{i}
$$

\subsection{Functions}

The function to be optimized, the LCOE, is an aggregation of two criteria, namely, the total investment cost and the thermal energy collected by the field.

The investment cost $C$ takes into account the investment in solar power plant equipment (tower, receivers and heliostats). Hence it depends on the receiver variable $\Theta$, and the number of heliostats in the field $|\mathcal{S}|$, as follows:

$$
C(\Theta,|\mathcal{S}|)=\beta_{1}\left(\max _{i}\left\{h_{i}\right\}+\kappa\right)^{\sigma}+\beta_{2} \pi \sum_{i=1}^{3} r_{i}^{2}+c_{F}+c|\mathcal{S}|,
$$




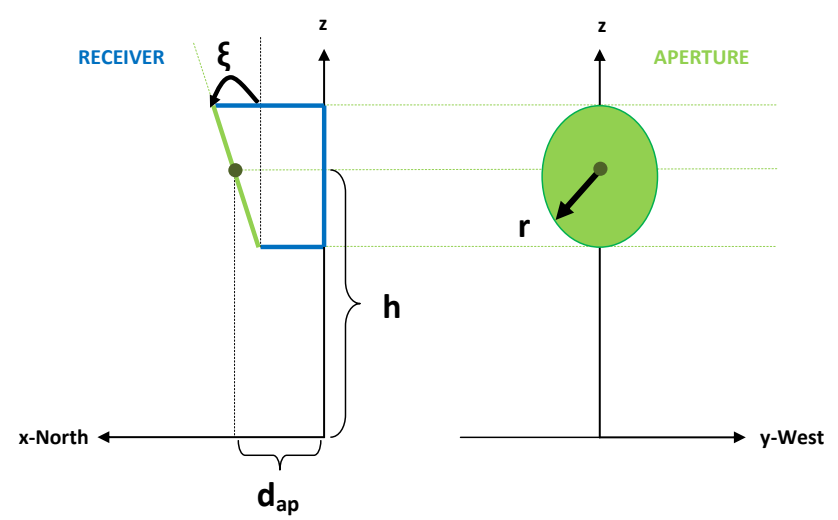

(a) $h, \xi$ angle and $r$ (front-lateral)

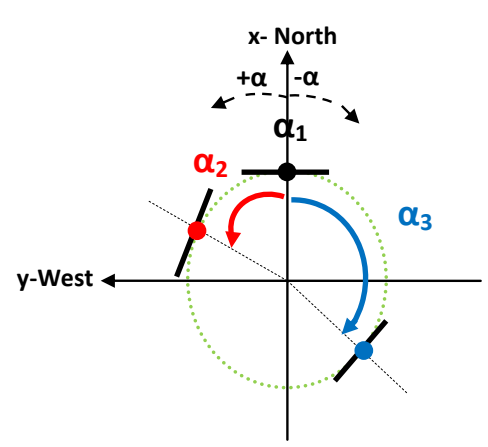

(b) $\alpha$ angle (top)

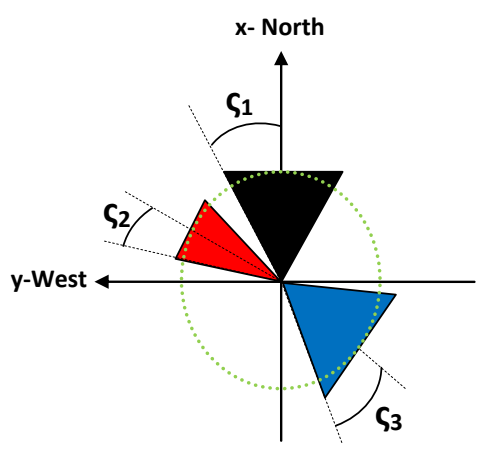

(c) $\varsigma$ angle (top)

Figure 1: Receivers variables 
where $\beta_{1}$ and $\beta_{2}$ are empirical constants with values in $(0,1), \kappa$ and $\sigma$ are positive and given by appropriate physical considerations and $c$ denotes the cost per heliostat. For simplicity, the cost associated to the land (purchasing and preparing) is considered fixed and denoted by $c_{F}$.

The thermal annual energy $E$ collected by the field into the receiver takes the form:

$$
E(\Theta, \mathcal{S})=\int_{0}^{T} \Pi_{t}(\Theta, \mathcal{S}) d t-\gamma_{1},
$$

where $t$ denotes the time instant, $\Pi_{t}$ is the outlet thermal power collected at time $t$, calculated as in $[5,12,16,17,18]$, and $\gamma_{1}$ is a constant that mesures the fixed energy losses related with the whole system.

\section{Problem Statement}

Our goal is to design an SPT system with multiple receivers technology in order to minimize the LCOE of the system. Usually, when designing an SPT system, a fixed time instant is used to size the plant, as explained in [12, 28]. This time instant is known in the literature as the design point, denoted by $T_{d}$, where a minimal power for each receiver is required, that is:

$$
\Pi_{T_{d}}\left(\Theta_{i}, \mathcal{S}\right) \geq \Pi_{i}^{0} \quad \forall i=1,2,3 .
$$

Including these constraints, the optimization problem can be written as follows:

$$
(\mathcal{P}) \begin{cases}\min _{\Theta, \mathcal{S}} & F(\Theta, \mathcal{S})=C(\Theta,|\mathcal{S}|) / E(\Theta, \mathcal{S}) \\ \text { subject to } & \Theta \in \Theta \\ & \mathcal{S} \in \mathscr{S} \\ & \Pi_{T_{d}}\left(\Theta_{i}, \mathcal{S}\right) \geq \Pi_{i}^{0} \quad \forall i=1,2,3 .\end{cases}
$$

The characteristics of the objective function are challenging: nonconvex, black-box type, computationally expensive and the number of variables (thousands of heliostats in commercial plants, see [10]), making this problem difficult to solve.

The optimization problem $(\mathcal{P})$ has two blocks of decision variables, namely those related to the design of the receiver $\Theta$, and those related to the field layout, $\mathcal{S}$. In order to handle the problem, as we did with a single receiver in [12], we are going to separate $(\mathcal{P})$ in two subproblems: the Multiple Receivers Optimization and the Field Optimization.

The Multiple Receivers Optimization problem, denoted by $\left(\mathcal{P}_{\mathcal{S}}\right)$ and given below, describes the optimization of the multiple receivers when the field of heliostats is fixed. 


$$
\left(\mathcal{P}_{\mathcal{S}}\right) \quad \mathcal{S} \text { fixed } \begin{cases}\min _{\Theta} & F(\Theta, \mathcal{S}) \\ \text { subject to } & \Theta \in \Theta \\ & \Pi_{T_{d}}\left(\Theta_{i}, \mathcal{S}\right) \geq \Pi_{i}^{0} \quad \forall i=1,2,3 .\end{cases}
$$

The second subproblem, the Field Optimization problem denoted by $\left(\mathcal{P}_{\Theta}\right)$, describes the field optimization for a fixed multiple receivers configuration:

$$
\left(\mathcal{P}_{\Theta}\right) \quad \Theta \text { fixed } \begin{cases}\min _{\mathcal{S}} & F(\Theta, \mathcal{S}) \\ \text { subject to } & \mathcal{S} \in \mathscr{S} \\ & \Pi_{T_{d}}\left(\Theta_{i}, \mathcal{S}\right) \geq \Pi_{i}^{0} \quad \forall i=1,2,3 .\end{cases}
$$

Observe that the cardinality of $\mathcal{S}$ is not fixed in advance in problems $(\mathcal{P})$ and $\left(\mathcal{P}_{\Theta}\right)$, thus we cannot express them as standard optimization problems in fixed dimension.

In the following subsections we present strategies devised to solve problems $\left(\mathcal{P}_{\Theta}\right)$ and $\left(\mathcal{P}_{\mathcal{S}}\right)$ independently. Once both subproblems are solved, we apply an alternating algorithm to obtain a solution of the main problem $(\mathcal{P})$. This alternating algorithm sequentially optimizes the field for the receivers given configuration and, then, the receivers are optimized for the obtained field. The process is repeated until no improvement in the objective function is reached or the difference between two consecutive iterations is irrelevant, that is $\left\|F\left(\Theta^{k}, \mathcal{S}^{k}\right)-F\left(\Theta^{k+1}, \mathcal{S}^{k+1}\right)\right\| \leq \epsilon_{0}$ for a given $\epsilon_{0}$. See [12], for further details on the application of this approach to the single receiver case.

\subsection{Multiple Receivers Optimization $\left(\mathcal{P}_{\mathcal{S}}\right)$}

In order to design a multiple receivers configuration we propose to optimize them sequentially. We say that the multiple receivers optimization algorithm performs an iteration once all the receivers have been modified. The algorithm stops after an iteration when no improvement in the objective function is found or the difference between the obtained configuration and the previous one is insignificant, i.e., $\left\|\Theta^{k}-\Theta^{k+1}\right\|_{\infty} \leq \epsilon_{1}$ for a given $\epsilon_{1}$.

Individually, the optimization of each receiver is performed using the cyclic coordinate method, see [1]. The local search performed by the method at each variable is limited to the corresponding feasible interval given by the feasible set $\boldsymbol{\Theta}$, see (2). The power requirements are included in the objective function by penalizing the non-feasible solutions. At receiver $i$ the following stopping rule is applied: $\left\|\Theta_{i}^{k}-\Theta_{i}^{k+1}\right\|_{\infty} \leq \epsilon_{2}$ for a given $\epsilon_{2}$.

\subsection{Field Optimization $\left(\mathcal{P}_{\Theta}\right)$}

Locating heliostats amounts to deal with a difficult large-dimensional multimodal black-box optimization problem. This is why heuristic methods are applicable, which do not guarantee to find the globally optimal solution, but 
are fast and do not require much knowledge about the function itself. As customary in the literature, we impose that heliostats aiming different receivers can not be mixed. This way, we can divide the heliostat field optimization problem in two stages: the division of the feasible region $\Omega$ into three aiming regions and the location of heliostats within each aiming region obtaining the three separate fields $\mathcal{S}_{i}$. In the following, we detail the proposed procedure to solve problem $\left(\mathcal{P}_{\Theta}\right)$ under the previous assumptions.

Given a multiple receivers configuration $\Theta$, the field optimization procedure starts by discretizing the feasible region $\Omega$ in order to separate it into three regions of empty intersection. For each point $(x, y)$ in the feasible region $\Omega$, the energy generated by one single heliostat centered at $(x, y)$ and aiming at receiver $i$, for $i=1,2,3$, are calculated. Then, the optimal aiming per such point, if no other heliostats existed in the field, is identified as the receiver where the maximum thermal energy is collected, i.e. $\max _{i}\left\{E\left(\Theta_{i},\{(x, y)\}\right)\right\}$.

Since $\Omega$ is infinite, a finite grid is chosen, obtaining plots such as the one in Figure 2(a), yielding the energy generated, and Figure 2(b), yielding the split given by the optimal aiming: red, black and blue correspond with West, North and East receiver.

The figures used to illustrate the explanation are obtained after applying the proposed method to an annulus shaped feasible region $\Omega$ and the following multiple receivers configuration:

$$
\Theta=\left(\begin{array}{rrr}
100.5 & 100.5 & 100.5 \\
12.5 & 12.5 & 12.5 \\
0 & 90 & -90 \\
6.39 & 6.39 & 6.39
\end{array}\right)
$$

with units detailed in Table 3. An annulus shaped feasible region is considered since heliostats can not be placed neither near nor far away from the tower due to several limitations, for instance, security constraints.

As we already mentioned, the regions have not the same influence over the objective function (North region reaches better values if we are in the northern hemisphere, see Figure 2(a)) and also, different power requirements could be considered for each receiver. Therefore, the previous process may not lead to the best field configuration. Different weights are applied to the obtained energy values in order to give more or less priority to the northern region. That is, for each point $(x, y)$ and each weight $w$, the optimal aiming is calculated as the receiver where the maximum value is achieved, i.e. $\max \left\{w E\left(\Theta_{1},\{(x, y)\}\right), E\left(\Theta_{2},\{(x, y)\}\right), E\left(\Theta_{3},\{(x, y)\}\right)\right\}$.

Once one partition of $\Omega$ is obtained, the three sets of boundary points are identified. A polynomial fit is applied to each set of points. That is, three polynomial fits are applied to the following boundary points: North-West, NorthEast and West-East; obtaining $p_{w}, q_{w}$ and $s_{w}$ polynomials respectively. In the given example, the south region is fairly separated by the $x$ axis as can be seen in Figure 2(a).

Given the weight $w$ and following the previous notation, each aiming region 
is denoted by $\Omega_{i}^{w}$ and can be expressed as follows.

$$
\begin{aligned}
& \Omega_{1}^{w}=\left\{\begin{array}{ll}
(x, y) \in \Omega: & x \geq 0 \\
& q_{w}(x) \leq y \leq p_{w}(x)
\end{array}\right\},
\end{aligned}
$$

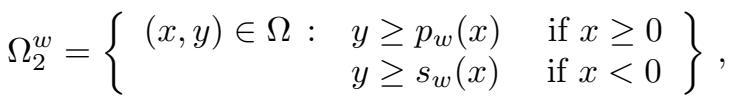

$$
\begin{aligned}
& \Omega_{3}^{w}=\left\{\begin{array}{lll}
(x, y) \in \Omega: & y \leq q_{w}(x) & \text { if } x \geq 0 \\
& y \leq s_{w}(x) & \text { if } x<0
\end{array}\right\} .
\end{aligned}
$$

As a first example, $w$ is set to 1 , i.e. no prioritized region is considered. After applying the procedure, $p_{1}, q_{1}$ and $s_{1}$ are obtained as shown in Figure 2(c), where the West-East polynomial corresponds to the $x$ axis.

If five different weights $w_{k} \in \mathbb{R}_{+}$are considered, for each weight we obtain the corresponding three polynomials $p_{w_{k}}, q_{w_{k}}$ and $s_{w_{k}}$, which define three different aiming regions. As can be seen in Figure 2(d), considering $w=1.010$, resp. $w=0.990$, more priority, resp. less priority, is given to the North region.

The problem is solved for the partitions of $\Omega$ corresponding to the various weights. At the end of the process the field layout which reaches the best LCOE value is selected as final solution.

Once we have calculated the three aiming regions for a given weight $w$, our second goal is to obtain the three heliostat field layouts, that is $\mathcal{S}_{i} \in \mathscr{S}_{i}^{w}$ where $\mathscr{S}_{i}^{w}=\mathcal{S} \cap \Omega_{i}^{w}$ for $i=1,2,3$. We start locating heliostats at the most favorable region, that is $\Omega_{1}^{w}$ if we are in the northern hemisphere. We obtain $\mathcal{S}_{1}$ solving problem $\left(\mathcal{P}_{\Theta}^{1}\right)$ given below:

$$
\left(\mathcal{P}_{\Theta}^{1}\right) \quad \Theta \text { fixed } \begin{cases}\min _{\mathcal{S}_{1}} & F\left(\Theta, \mathcal{S}_{1}\right) \\ \text { subject to } & \mathcal{S}_{1} \in \mathscr{S}_{1}^{w} \\ & \Pi_{T_{d}}\left(\Theta_{1}, \mathcal{S}_{1}\right) \geq \Pi_{0}^{1} .\end{cases}
$$

Once $\mathcal{S}_{1}$ is obtained, the procedure continues solving $\left(\mathcal{P}_{\Theta}^{2}\right)$ and $\left(\mathcal{P}_{\Theta}^{3}\right)$ simultaneously to obtain $\mathcal{S}_{2}$ and $\mathcal{S}_{3}$. Both problems are described below, for $i=2,3$ respectively:

$\left(\mathcal{P}_{\Theta}^{i}\right) \quad \Theta$ fixed $\begin{cases}\min _{\mathcal{S}_{i}} & F(\Theta, \mathcal{S}) \\ \text { subject to } & \mathcal{S}_{i} \in \mathscr{S}_{i}^{w} \\ & \left\|(x, y)-\left(x^{\prime}, y^{\prime}\right)\right\| \geq \delta \quad \forall(x, y) \in \mathcal{S}_{i},\left(x^{\prime}, y^{\prime}\right) \in \mathcal{S}_{1} \\ & \Pi_{T_{d}}\left(\Theta_{i}, \mathcal{S}_{i}\right) \geq \Pi_{0}^{i} .\end{cases}$

Note that in both problems, we include as constraints the heliostat positions from the subfield $\mathcal{S}_{1}$ already calculated. Collisions between heliostats must be avoided and the security distance must be respected by all the heliostats, including those located near the boundaries.

We apply a greedy-based algorithm to solve the heliostat location problems involved, see [12]. This Greedy Algorithm locates the heliostats one by one at the best position found in the selected region. At each step the shading and 


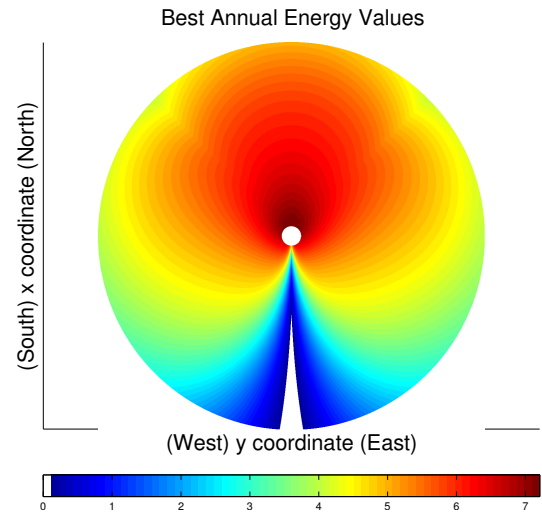

(a) Energy generated

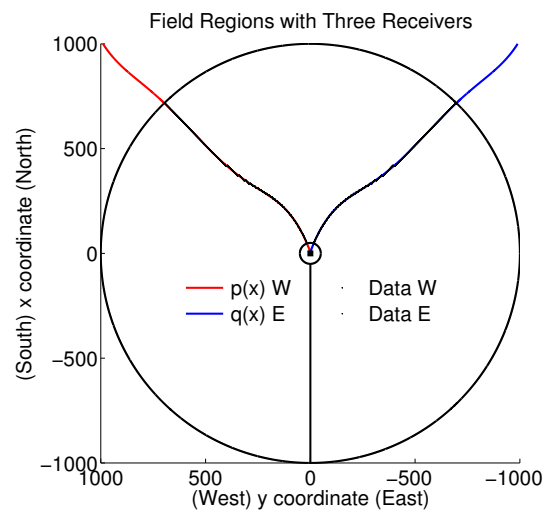

(c) Aiming regions for $w=1$

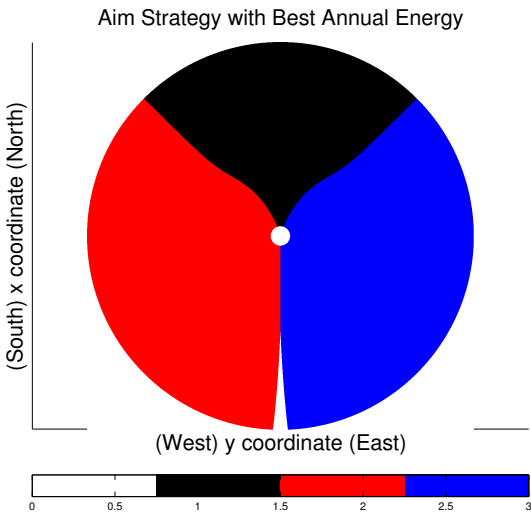

(b) Optimal aiming

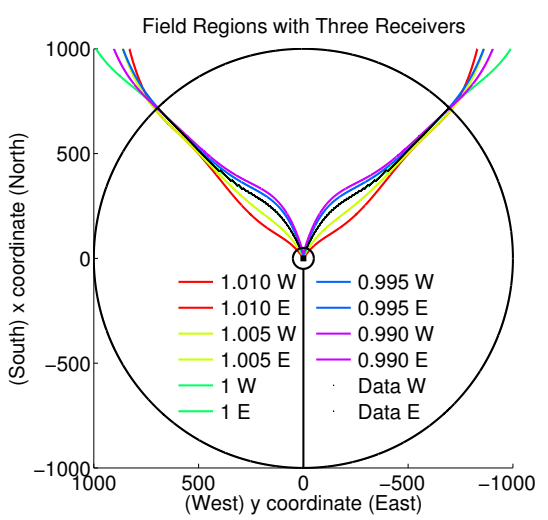

(d) Aiming regions for several $w$

Figure 2: Aiming regions calculation 
blocking effects are modified taking into account the heliostats already located. The heliostat location method is described in Algorithm 1, where $\left(\mathcal{P}_{\Theta}^{i}\right)^{k}$ denotes the location of one heliostat at aiming region $i$ where there are already $k-1$ heliostats located in the field. The method has two phases. In the first phase, called Requirement Phase, heliostats are sequentially added, and the process is stopped when the power requirement of the corresponding receiver is reached.

The Requirement Phase ends with a feasible field, in which the power at the design point is attained. To minimize the LCOE of the whole field, we continue locating heliostats in a second phase, called Completion Phase, even though we exceed the minimal power requirements. Applying again the Greedy Algorithm at each of the three subfields we obtain three new heliostat positions: $\left\{\left(x_{i}, y_{i}\right)\right\}_{i=1}^{3}$. The heliostat positions selected to be part of the field is the one where the best LCOE value is achieved, that is the position $\left(x_{j}, y_{j}\right)$ where $\max _{i}\left\{E\left(\Theta, \mathcal{S} \cup\left\{\left(x_{i}, y_{i}\right)\right\}\right)\right\}$ is achieved. If none of the three new positions improves the LCOE of the system or any of the maximal power requirements $\Pi_{i}^{+}$is achieved (receiver security constraint), the algorithm stops and gives as solution the field obtained so far. Otherwise, the heliostat field is updated with the new position. 


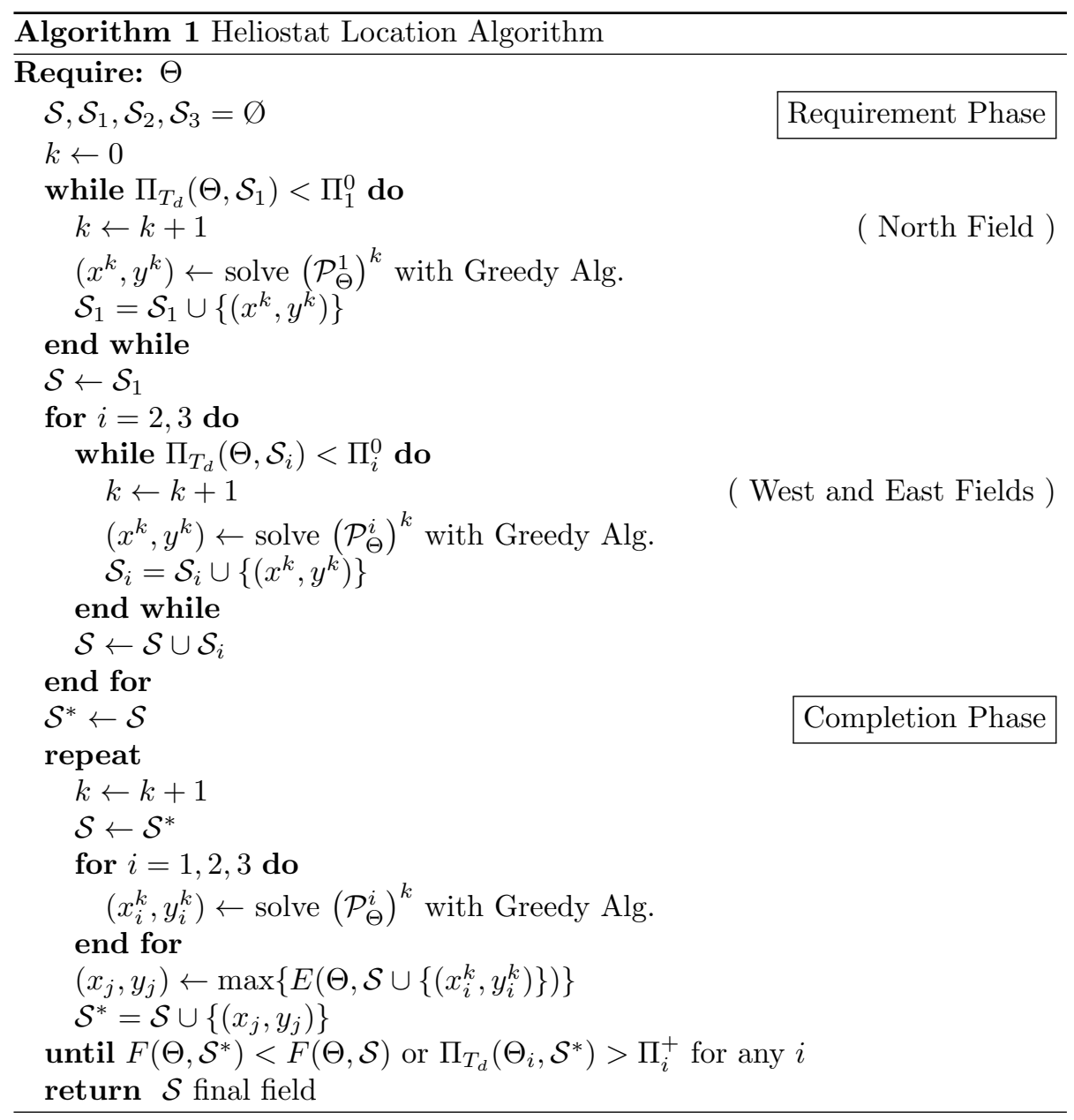

Following the example with weight $w=0.990$, after the Requirement Phase the algorithm gives as solution the field shown in Figure 3(a), where heliostats aiming different receivers are highlighted with different colors, and heliostats located at the Completion Phase are highlighted with white asterisks. The product of the efficiency coefficients (cosine, shading and blocking, interception and atmospheric) for each heliostat at different time instants are shown in Figure 3(b)-3(d).

\section{Results}

The SPT system is assumed to be placed at the same location of the reference plant called PS10, see [22]. In Table 1, all the fixed parameters are detailed. The lack of results available in the literature in this multiple receivers approach 


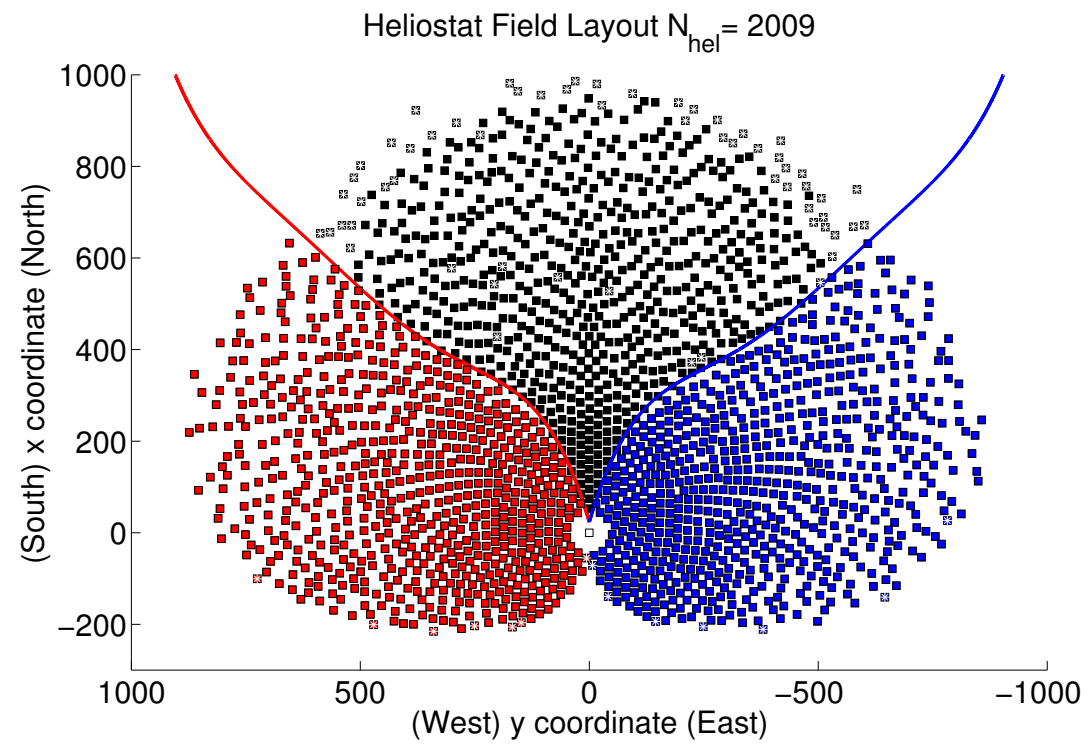

(a) $\mathcal{S}^{0}$ with $w=0.990$ and $\Theta^{0}$ fixed

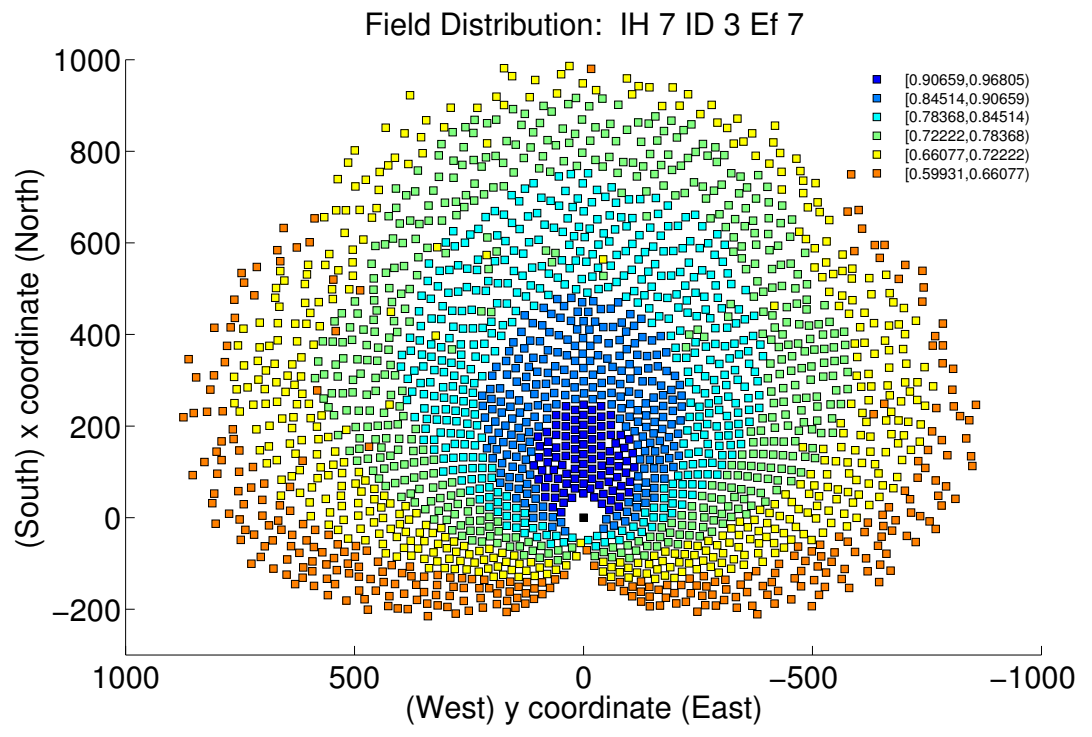

(b) $\mathcal{S}^{0}$ (21st March $12 \mathrm{~h}$ ) 


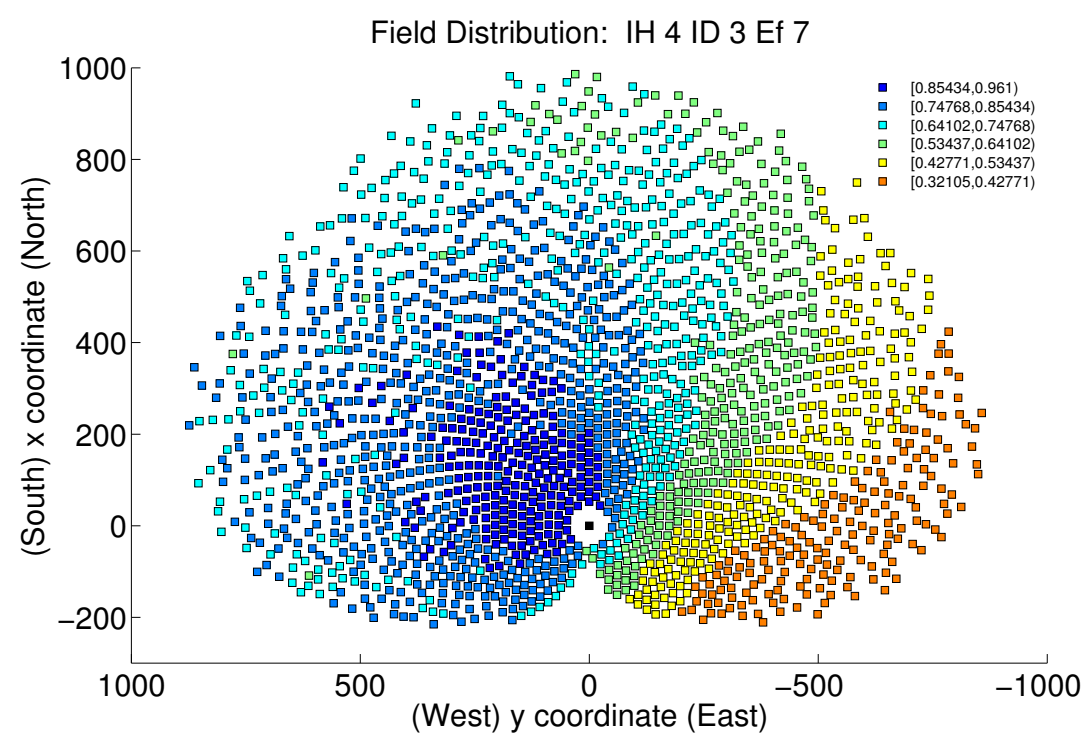

(c) $\mathcal{S}^{0}$ (21st March $\left.09 \mathrm{~h}\right)$

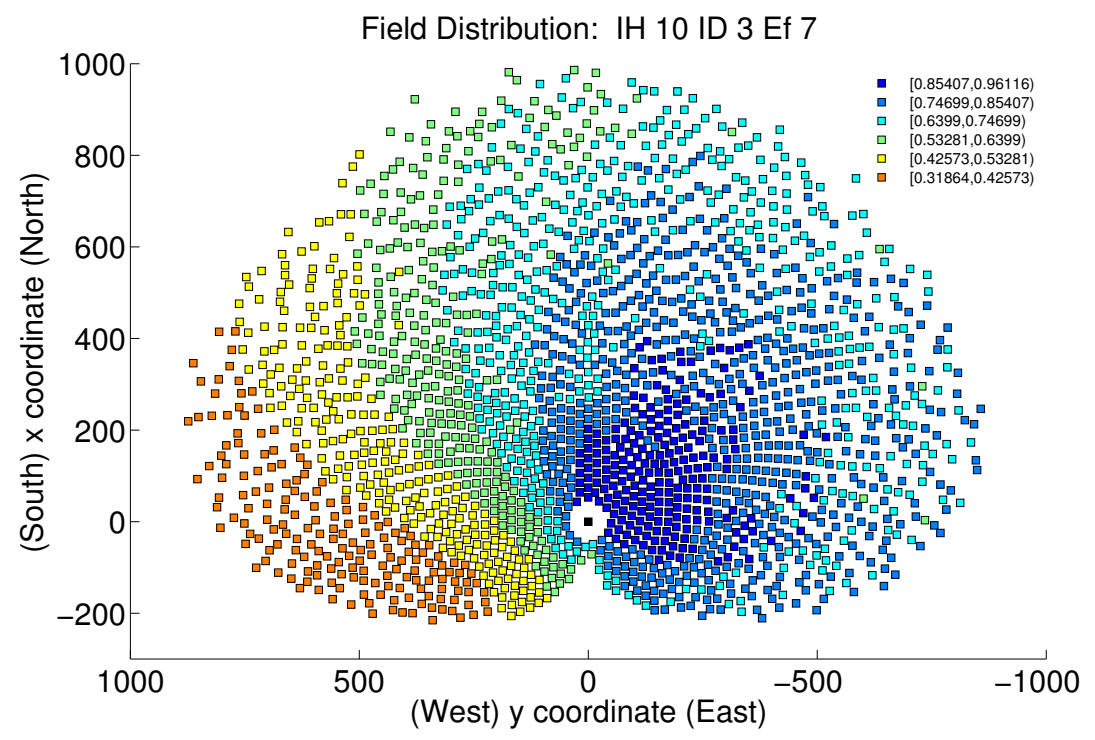

(d) $\mathcal{S}^{0}$ (21st March $\left.15 \mathrm{~h}\right)$

Figure 3: Heliostat Field $\mathcal{S}^{0}$ and Efficiency Coefficient 
has made impossible to carry out a comparison of the obtained results with possible competitors.

\begin{tabular}{|c|c|c|}
\hline Parameter & Default value & Ref. \\
\hline \multicolumn{3}{|l|}{ Location and Time } \\
\hline $\begin{array}{l}\text { Site } \\
\text { Latitude } \\
\text { Longitude } \\
\text { Design Point } T_{d} \\
\text { Design direct normal irradiance DNI } \\
\text { DNI model }\end{array}$ & $\begin{array}{c}\text { Sanlúcar la Mayor (Seville) } \\
37^{\circ} 26^{\prime} \mathrm{N} \\
6^{\circ} 15^{\prime} \mathrm{W} \\
\text { March Day } 21 \text { Hour } 12 \\
823.9 \mathrm{~W} / \mathrm{m}^{2} \\
\text { cloudless sky }\end{array}$ & $\begin{array}{c}{[23]} \\
{[22]} \\
{[22]} \\
\text { assumed } \\
\text { assumed } \\
\text { assumed }\end{array}$ \\
\hline \multicolumn{3}{|l|}{ Heliostat } \\
\hline $\begin{array}{l}\text { Name } \\
\text { Width } \\
\text { Height } \\
\text { Optical height } z_{0} \\
\text { Minimal safety distance } \delta \\
\sigma_{\text {optical }}\end{array}$ & $\begin{array}{c}\text { Sanlucar } 120 \\
12.84 \mathrm{~m} \\
9.45 \mathrm{~m} \\
5.17 \mathrm{~m} \\
\text { heliostat diagonal+ds } \\
2.9 \mathrm{mrad} \\
\end{array}$ & $\begin{array}{l}{[22]} \\
{[22]} \\
{[22]} \\
{[23]} \\
{[8]} \\
{[22]} \\
\end{array}$ \\
\hline \multicolumn{3}{|l|}{ Field } \\
\hline $\begin{array}{l}\text { Slope } \\
\text { Shape } \\
\text { Minimum radius } \\
\text { Maximum radius }\end{array}$ & $\begin{array}{c}0^{\circ} \\
\text { annulus } \\
50 \mathrm{~m} \\
10^{3} \mathrm{~m}\end{array}$ & $\begin{array}{l}\text { assumed } \\
\text { assumed } \\
\text { assumed } \\
\text { assumed }\end{array}$ \\
\hline
\end{tabular}

Table 1: Parameter Values

We detail in this section the iterations performed by the proposed alternating algorithm. As detailed in Section 3.2-Figure 2(d), we have considered five different weights in previous experiments and the best results were found with $w=0.990$. We maintain this value constant, and we apply the Alternating algorithm taking as initial solution $\Theta^{0}$, detailed in Table 3. The minimal and maximal power requirements are set to $\Pi^{0}=38.27 \mathrm{MWth}$ and $\Pi^{+}=40.18$ $M W t h$ respectively and equal for the three receivers.

The Alternating algorithm performs three complete iterations and stops when $\left\|F\left(\Theta^{1}, \mathcal{S}^{1}\right)-F\left(\Theta^{2}, \mathcal{S}^{2}\right)\right\|<\epsilon_{0}$, with $\epsilon_{0}=0.001$. The three heliostat fields obtained during the process, $\mathcal{S}^{0}, \mathcal{S}^{1}$ and $\mathcal{S}^{2}$, are shown in Figures 3(a)-4(a)4 (b), respectively. Regarding the multiple receivers optimization algorithm, the stopping parameters are set as $\epsilon_{1}=\epsilon_{2}=0.1$.

As shown in Table 3 and Figure 5, applying the algorithm a reduction on the LCOE value is achieved. Note that the label enumeration corresponds with the configurations detailed in Table 3 (column 2) and that heliostats aiming different receivers are highlighted with different colors.

During the optimization process, the aperture radiuses are reduced and the receivers positions are modified, unlike the receivers height, that remains approximately constant. The aiming regions, location and number of heliostats in the different fields have also been modified by the algorithm according to the receivers modifications. The final solution is configuration number 3 which 
corresponds with $\left(\Theta^{1}, \mathcal{S}^{1}\right)$ where the minimum LCOE value is achieved.

Regarding the obtained heliostat field layouts, some irregularities appear at the boundaries. The shape of the final field could be smoothed by using, for instance, a nonrestricted refinement method [8] or selecting continuous piecewise linear polynomials to better adapt the boundaries discontinuities.

\begin{tabular}{r|rccrc}
\hline Step & & $h[\mathrm{~m}]$ & $\xi$ [grad] & $\alpha$ [grad] & $r[\mathrm{~m}]$ \\
\hline \multirow{3}{*}{$\Theta^{0}$} & $\Theta_{1}$ & 100.50 & 12.50 & 0 & 6.39 \\
& $\Theta_{2}$ & 100.50 & 12.50 & 90 & 6.39 \\
& $\Theta_{3}$ & 100.50 & 12.50 & -90 & 6.39 \\
\hline \multirow{3}{*}{$\Theta^{1}$} & $\Theta_{1}$ & 100.53 & 8.72 & -0.81 & 4.83 \\
& $\Theta_{2}$ & 100.50 & 17.24 & 80.94 & 4.44 \\
& $\Theta_{3}$ & 100.50 & 17.96 & -81.32 & 4.48 \\
\hline \multirow{3}{*}{$\Theta^{2}$} & $\Theta_{1}$ & 100.50 & 10.71 & -0.26 & 4.44 \\
& $\Theta_{2}$ & 100.50 & 17.50 & 75.41 & 4.11 \\
& $\Theta_{3}$ & 100.50 & 17.43 & -76.09 & 4.11 \\
\hline
\end{tabular}

Table 2: Alternating Algorithm Results: Receiver

\begin{tabular}{c|c|ccccc}
\hline Step & $\mathrm{Pb}$ & $|\mathcal{S}|$ & $\Pi_{T_{d}}[\mathrm{MWth}]$ & $E[\mathrm{GWHth}]$ & $C[\mathrm{M} €]$ & $C / E$ \\
\hline$k=0$ & $1:\left(\Theta^{0}, \mathcal{S}^{0}\right)$ & 2009 & 118.7550 & 326.83 & 5.9984 & 0.01835 \\
\hline \multirow{2}{*}{$k=1$} & $2:\left(\Theta^{1}, \mathcal{S}^{0}\right)$ & 2009 & 112.0731 & 310.62 & 5.3916 & 0.01736 \\
& $3:\left(\Theta^{1}, \mathcal{S}^{1}\right)$ & 2033 & 115.0178 & 314.55 & 5.4445 & 0.01731 \\
\hline \multirow{3}{*}{$k=2$} & $4:\left(\Theta^{2}, \mathcal{S}^{1}\right)$ & 2033 & 110.4583 & 306.45 & 5.3443 & 0.01744 \\
& $5:\left(\Theta^{2}, \mathcal{S}^{2}\right)$ & 2084 & 114.8432 & 312.30 & 5.4567 & 0.01747 \\
\hline
\end{tabular}

Table 3: Alternating Algorithm Results: Configurations

\section{Conclusions and further work}

In this article, a method to design multiple receivers SPT systems is proposed, where the receivers and the heliostat field are simultaneously optimized. The method identifies the so-called aiming areas (regions of points with the same aiming strategy). The boundaries of the aiming areas are calculated by polynomial approximation, and embedded in a pattern-free procedure, called Greedy Algorithm [12], applied to locating the heliostats.

Several extensions of this work are possible, in some cases with not much effort. Throughout the paper we have considered independent power threshold levels for the different receivers, each measured at one (common) design point. It is straightforward to extend our approach to the case in which overall power threshold levels, or multiple design points are considered. 


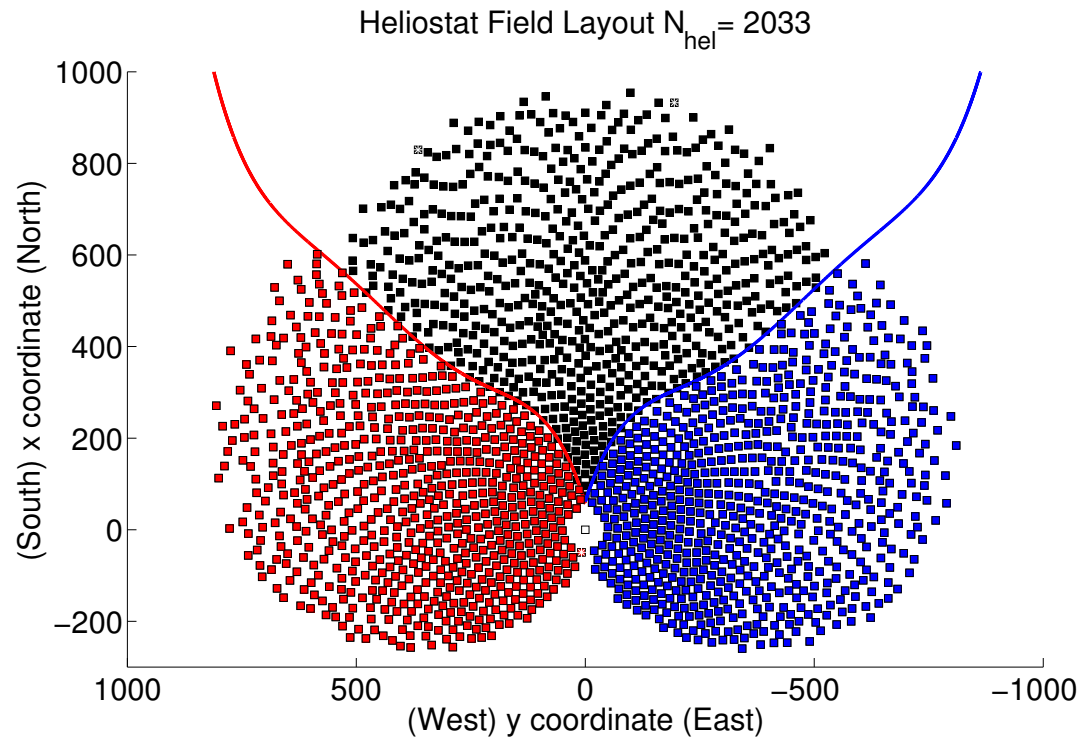

(a) $\mathcal{S}^{1}\left(\Theta^{1}\right.$ fixed) Final Field

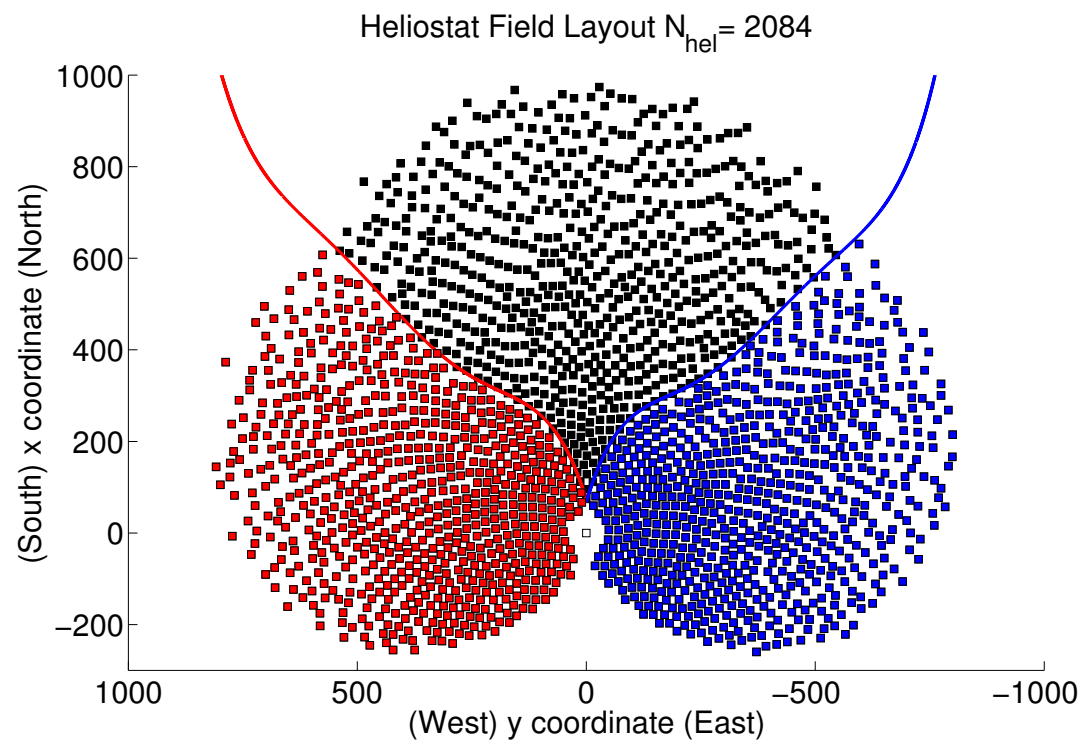

(b) $\mathcal{S}^{2}\left(\Theta^{2}\right.$ fixed $)$

Figure 4: Heliostat Fields 


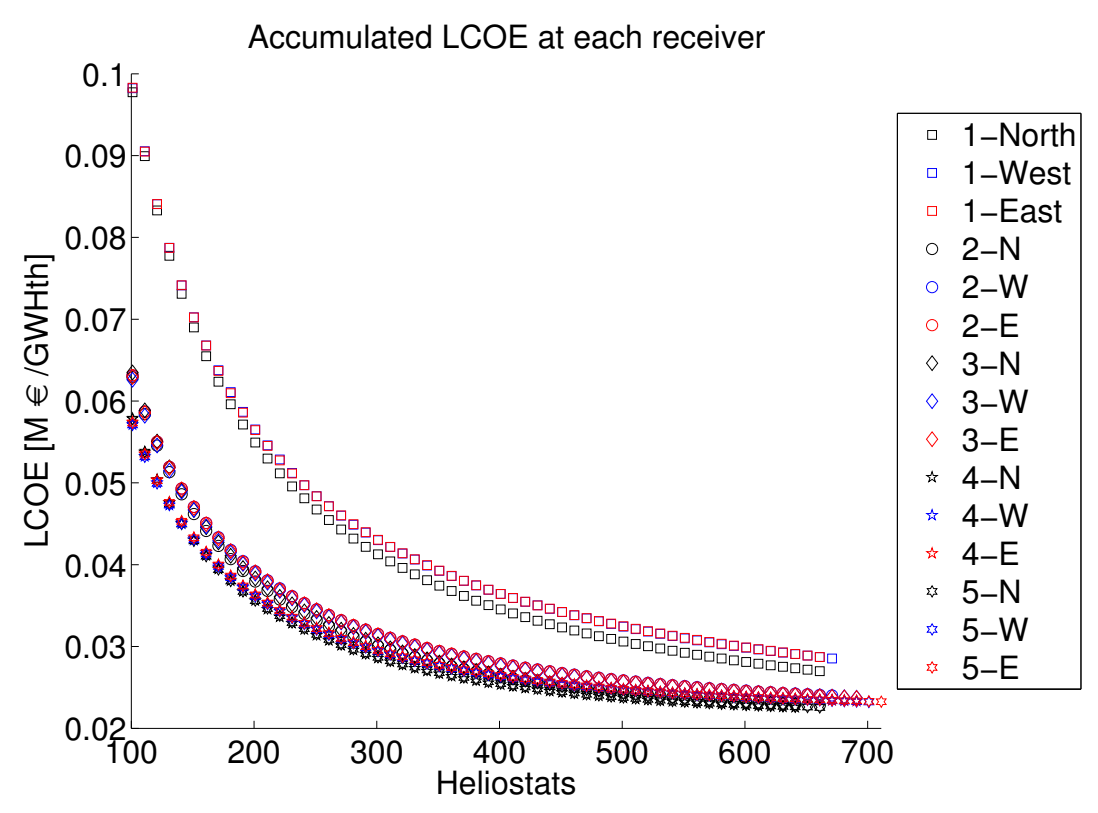

Figure 5: Alternating Process: LCOE (M€/GWHth)

The calculation of the optimal number of receivers and the design of an aiming strategy are not addressed in this work. We assume a homogeneous flux distribution, see [9]. However, differences in flux density will occur over the absorber area, and the distribution will also vary during the day and the seasons. An appropriate control is required to adapt the mass flow in the absorber to the solar distribution. The design of an aiming strategy is a continuous nonlinear constrained black-box optimization problem of very large dimensions as pointed out in [3], which deserves further analysis, see for instance [19].

The field has been designed so that one natural performance, namely, the LCOE, is optimized. However, solving the optimization problem with various objective functions is also possible. See for instance [36] for a multi-objective thermoeconomic optimization approach with one single receiver.

Multi-Towers configurations are also an interesting innovation in SPT systems, see [30]. The calculation of the optimal towers positions and the aiming strategies to be applied are the major additional difficulties, which may be addressed by a repeated application of the methods presented here.

\section{Acknowledgements}

This research has been mainly supported by Abengoa Solar N.T. and Institute of Mathematics of University of Seville (IMUS), through the research contract "CapTorSol". The authors would also like to acknowledge the support from the Government of Spain (Grants MTM2010-15992, MTM2012-36136, 
MTM2013-41286-P), Andalucía (Grant P11-FQM-7603) and EU COST Action TD1207.

\section{References}

[1] Bazaraa, M. S., Sherali, H. D., \& Shetty, C. M. (2006). Nonlinear Programming: Theory and Algorithms. John Wiley and Sons, Hoboken, New Jersey.

[2] Behar, O., Khellaf, A., \& Mohammedi, K. (2013). A review of studies on central receiver solar thermal power plants. Renewable and Sustainable Energy Reviews, 23, 12-39.

[3] Belhomme, B., Pitz-Paal, R., \& Schwarzbözl, P. (2014). Optimization of heliostat aim point selection for central receiver systems based on the ant colony optimization metaheuristic. Journal of Solar Energy Engineering, $136,1-7$.

[4] Ben-Zvi, R., Epstein, M., \& Segal, A. (2012). Simulation of an integrated steam generator for solar tower. Solar Energy, 86, 578-592.

[5] Biggs, F., \& Vittitoe, C. (1976). The HELIOS model for the optical behavior of reflecting solar concentrators. Technical Report SAND760347 Sandia National Labs. URL: http://prod.sandia.gov/techlib/ access-control.cgi/1976/760347.pdf.

[6] BrightSource (2014). Ivanpah Solar Electric Generating System. Technical Report BrightSource. URL: www.brightsourceenergy.com/ stuff/contentmgr/files/0/3eac1a9fed7f13fe4006aaab8c088277/ attachment/ivanpah_white_paper_0414.pdf.

[7] Broze, J. G., Ranade, S., \& Prengle, H. W. a. (1985). An approximate model for sizing and costing a solar thermal collector-central receiver system. Solar Energy, 34, 341-350.

[8] Buck, R. (2014). Heliostat field layout improvement by nonrestricted refinement. Journal of Solar Energy Engineering, 136, 1-6.

[9] Buck, R., Barth, C., Eck, M., \& Steinmann, W. (2006). Dual receiver concept for solar tower. Solar Energy, 80, 1249-1254.

[10] Burgaleta, J. I., Arias, S., \& Ramirez, D. (2011). GEMASOLAR, the first tower thermosolar commercial plant with molten salt storage. In Proceedings of SolarPaces 2011.

[11] Carrizosa, E., Domínguez-Bravo, C., Fernández-Cara, E., \& Quero, M. (2014). An Optimization Approach to the Design of Multi-Size Heliostat fields. Technical Report IMUS. URL: www.optimization-online.org/ DB_HTML/2014/05/4372.html. 
[12] Carrizosa, E., Domínguez-Bravo, C., Fernández-Cara, E., \& Quero, M. (2015). A heuristic method for simultaneous tower and pattern-free field optimization on solar power systems. Computers $\&$ Operations Research, $57,109-122$.

[13] Collado, F. J. (2008). Quick evaluation of the annual heliostat field efficiency. Solar Energy, 82, 379-384.

[14] Collado, F. J., \& Guallar, J. (2012). Campo: Generation of regular heliostat fields. Renewable Energy, 46, 49-59.

[15] Collado, F. J., \& Guallar, J. (2013). A review of optimized design layouts for solar power tower plants with campo code. Renewable and Sustainable Energy Reviews, 20, 142-154.

[16] Collado, F. J., \& Turégano, J. (1986). An analytic function for the flux density due to sunlight reflected from a heliostat. Solar Energy, 37, 215234.

[17] Collado, F. J., \& Turégano, J. (1989). Calculation of the annual thermal energy supplied by a defined heliostat field. Solar Energy, 42, 149-165.

[18] Crespo, L., \& Ramos, F. (2009). NSPOC: A New Powerful Tool for Heliostat Field Layout and Receiver Geometry Optimizations. In Proceedings of SolarPaces 2009.

[19] Kribus, A., Vishnevetsky, I., Yogev, A., \& Rubinov, T. (2004). Closed loop control of heliostats. Energy, 29, 905-913.

[20] Leonardi, E., \& D'Aguanno, B. (2011). Crs4-2: A numerical code for the calculation of the solar power collected in a central receiver system. Energy, $36,4828-4837$.

[21] Mills, D. R. (2004). Advances in solar thermal electricity technology. Solar Energy, 76, 19-31.

[22] Noone, C. J., Torrilhon, M., \& Mitsos, A. (2012). Heliostat field optimization: A new computationally efficient model and biomimetic layout. Solar Energy, 86, 792-803.

[23] Osuna, R., Fernández, V., Romero, S., Romero, M., \& Sánchez, M. (2004). PS10: a 11.0-MWe Solar Tower Power Plant with Satured Steam Receiver. In Proceedings of SolarPaces 2004. Editors: C. Ramos and J. Huacuz.

[24] Ramos, A., \& Ramos, F. (2012). Strategies in Tower Solar Power Plant optimization. Solar Energy, 86, 2536-2548.

[25] Romero, M., Buck, R., \& Pacheco, J. E. (2002). An update on Solar Central Receiver Systems, Projects and Technologies. Solar Energy Engineering, 124, 98-109. 
[26] Rovira, A., Montes, M. J., Valdes, M., \& Martínez-Val, J. M. (2011). Energy management in solar thermal power plants with double thermal storage system and subdivided solar field. Applied Energy, 88, 4055-4066.

[27] Sánchez-González, A., \& Santana, D. (2014). Solar flux distribution on central receivers: A projection method from analytic function. Renewable Energy, 74, 576-587.

[28] Sanz-Bermejo, J., Gallardo-Natividad, V., Gonzalez-Aguilar, J., \& Romero, M. (2014). Comparative system performance analysis of direct steam generation central receiver solar thermal power plants in megawatt range. Journal of Solar Energy Engineering, 136, 1-9.

[29] Schmitz, M., Schwarzbözl, P., Buck, R., \& Pitz-Paal, R. (2006). Assesment of the potencial improvement due to multiple apertures in central receiver systems with secondary concentrators. Solar Energy, 80, 111-120.

[30] Schramek, P., Mills, D. R., Stein, W., \& Le Livre, P. (2009). Design of the Heliostat Field of the CSIRO Solar Tower. Journal of Solar Energy Engineering, 131, 024505.

[31] Segal, A., \& Epstein, M. (1999). Comparative performances of tower-top and tower-reflector central solar receivers. Solar Energy, 65, 207-226.

[32] Segal, A., \& Teufel, E. (2012). Optimum layout of heliostat field when the tower-top receiver is provided with secondary concentrators. Technical Report Weizmann Institute of Science (SFERA). URL: http://sfera.sollab.eu/downloads/JRA/WP13/R13. 3b_SFERA_WP13T2_Opt_HelioField_Secondary_TopReceiver.pdf.

[33] SolarPACES-NREL (2011). Gemasolar Thermosolar Plant. Technical Report Sener. URL: www.nrel.gov/csp/solarpaces/project_detail.cfm/ projectID $=40$.

[34] SolarPACES-NREL (2014). Ivanpah Solar Electric Generating System. Technical Report BrightSource Energy. URL: www.nrel.gov/csp/ solarpaces/project_detail.cfm/projectID=62.

[35] SolarPACES-NREL (2014). Khi Solar One. Technical Report Abengoa Solar. URL: www.nrel.gov/csp/solarpaces/project_detail.cfm/ projectID $=244$.

[36] Spelling, J., Favrat, D., Martin, A., \& Augsburger, G. (2012). Thermoeconomic optimization of a combined-cycle solar tower power plant. Energy, $41,113-120$. 